\title{
Rotor Resistance Online Identification of Vector Controlled Induction Motor Based on Neural Network
}

\author{
Bo Fan, ${ }^{1}$ Zhixin Yang, ${ }^{2}$ Wei Xu, ${ }^{3}$ and Xianbo Wang ${ }^{2}$ \\ ${ }^{1}$ Information Engineering College, Henan University of Science and Technology, Luoyang 471023, China \\ ${ }^{2}$ Department of Electromechanical Engineering, Faculty of Science and Technology, University of Macau, Macau \\ ${ }^{3}$ Faculty of Business Administration, University of Macau, Macau \\ Correspondence should be addressed to Zhixin Yang; zxyang@umac.mo
}

Received 7 May 2014; Accepted 3 August 2014; Published 25 September 2014

Academic Editor: Chengjin Zhang

Copyright (C) 2014 Bo Fan et al. This is an open access article distributed under the Creative Commons Attribution License, which permits unrestricted use, distribution, and reproduction in any medium, provided the original work is properly cited.

\begin{abstract}
Rotor resistance identification has been well recognized as one of the most critical factors affecting the theoretical study and applications of AC motor's control for high performance variable frequency speed adjustment. This paper proposes a novel model for rotor resistance parameters identification based on Elman neural networks. Elman recurrent neural network is capable of performing nonlinear function approximation and possesses the ability of time-variable characteristic adaptation. Those influencing factors of specified parameter are analyzed, respectively, and various work states are covered to ensure the completeness of the training samples. Through signal preprocessing on samples and training dataset, different input parameters identifications with one network are compared and analyzed. The trained Elman neural network, applied in the identification model, is able to efficiently predict the rotor resistance in high accuracy. The simulation and experimental results show that the proposed method owns extensive adaptability and performs very well in its application to vector controlled induction motor. This identification method is able to enhance the performance of induction motor's variable-frequency speed regulation.
\end{abstract}

\section{Introduction}

The key on vector control for induction motor lies in the magnetic field orientation, but one of the important factors affecting the field orientation is the accuracy of rotor parameters. While an AC motor is running, its parameters may change with the influence of inner and outer conditions. The changes of the motor temperature and slip frequency can affect rotor resistance value; it may increase $50 \%$ with the temperature rise and enhance a few times with the skin effect if the rotor current frequency is high. Also, the rotor inductance will change with magnetic saturation. It is a nonlinear relationship between this change value and the magnetic saturation degree; then, the rotor time constant will vary with the conditions. When the rotor time constant deviates from actual value largely, the decoupling conditions of the flux and torque control are destroyed; thus, the dynamic performance of the system depends on the rotor resistance's online identification and adjustment $[1,2]$. In order to improve the performance of induction motor vector control system, it is necessary to introduce the motor parameter online identification.

There are three main categories on the online parameter identification: spectrum analysis technology, the observerbased technology, and the model reference adaptive identification technology. Spectrum analysis adopts the motor's response of the injected signal or the harmonic characteristic information on the normal voltage and current signal. The required parameters can be obtained by the spectrum analysis on the stator current or voltage signal. As mentioned in [3], based on $d-q$ model in frequency domain, the $q$ axis component into the negative sequence signal remains zero, so that the motor torque is not disturbed. The fast Fourier transform is used to analyze the fundamental component of current and voltage as well as the samples spectral values, and the results were used to determine the parameters of the motor.

In the observer-based technology, the motor's parameters are processed as the system extended state. The main methods are the extension of Kalman filter (EKF) and the extended 
Luenberger observer (ELO). With the condition of the induction motor normal operating, the extended motor model and the EKF method on the motor parameter estimation are described in [4]. This method requires the motor end signal and rotor speed measurement. Reference [5] adopts the wideband harmonic contained in the PWM inverter output voltage to estimate the rotor time constant with EKF algorithm. The extended Luenberger observer for estimating the critical state and parameters in the motor is described in [6-8]. The main problem of EKF and ELO is the calculation of strength. The more the numbers of parameters estimation of the expansion are, the more the strength calculation increases rapidly.

The characteristics of the model reference adaptive identification technique are simple, but its accuracy depends on the accuracy of the model. The method based on reactive power is not sensitive to the variation of stator resistance, which is the most common model reference adaptive control method $[9,10]$. In order to reduce the change of other parameters of the sensitivity, it is better to estimate some other parameters during the adaptive adjustment. When the condition of the magnetizing inductance variation with the degree of saturation of the motor is considered, the identification accuracy of the stator and the rotor resistance are further improved [11-13].

Additionally, online identification of rotor resistance for vector controlled induction motor has been focused on more and more. A time-varying parameter estimation algorithm is presented in [14], which is simple and easy for online estimation of the rotor resistance for induction motor with the rapidly convergence in spite of measurement noise, discretization effects, parameter uncertainties, and modeling inaccuracies. The parametric model is deduced by the transfer function based on the stationary state, and a calculating is built to a plurality of motor parameters calculation [15]. A fuzzy logic MRAS method with realization forms is proposed in [16], in which the reference and adaptive models are deduced from different models, and they all depend on Popov's hyperstatic theory, but the acquisition of reference model always has difficulties. A MRAS system composed of rotor voltage and current model is provided by [17], in which a PI regulator is used and also suitable for the system with sensor only.

Artificial neural network is increasingly used for parameter identification in recent years. It is illustrated systematically that the neural networks are applied to electrical drive systems in [18] and give valuable instruction of their online and offline realization. The implementation of rotor resistance online identification with $\mathrm{BP}$ neural network is explored in [19], which provides an effective method. A parameter identification method based on Hopfield neural network is presented in [20], which also gives sufficient condition of correct identification with sensor signal delay. Besides, there are also neural network schemes combined with wavelet transformation [21] and Kalman filter method [22]. These studies proved neural network's applicability to motor parameter identification theoretically, with simulation and in practice.
The model of rotor resistance identification based on Elman neural network is proposed in this paper. Elman neural network is a kind of recurrent network, which can approximate any function with any preciseness theoretically. A context layer to the forward network is added as a delay factor to memorize history state. So this model has the abilities of adapting to time-variable characteristic, reflecting the dynamic characteristics of system directly, and the stronger calculation. With the simulation and experiments, Elman neural network is proved to be suitable to resolve the problem of motor parameter identification.

\section{Models of Induction Motor}

The equations of induction motor in the 3-phase static coordinate, after $3 / 2$ transform, can be obtained in the 2phase stationary coordinate system of equations; also after $2 s / 2 r$ transformation, it can be measured in 2-phase rotating coordinate system on any mathematical model.

At first, the mathematics model in the 2-phase synchronous rotating coordinate system of the induction motor is presented. The 2-phase synchronous rotating coordinate system is a special case of two arbitrary rotation coordinates rotating in synchronous speed, also known as the MT coordinate system. Mathematical model equations with this system of the induction motor are shown as follows.

Voltage equation is given by

$$
\begin{aligned}
{\left[\begin{array}{c}
u_{s M} \\
u_{s T} \\
0 \\
0
\end{array}\right] } & {\left[\begin{array}{cccc}
R_{s}+L_{s} p & -\omega_{1} L_{s} & L_{m} p & -\omega_{1} L_{m} \\
\omega_{1} L_{s} & R_{s}+L_{s} p & \omega_{1} L_{m} & L_{m} p \\
L_{m} p & -\omega_{1} L_{m} & R_{r}+L_{r} p & -\omega_{s} L_{r} \\
\omega_{s} L_{m} & L_{m} p & \omega_{s} L_{r} & R_{r}+L_{r} p
\end{array}\right] } \\
& \times\left[\begin{array}{c}
i_{s M} \\
i_{s T} \\
i_{r M} \\
i_{r T}
\end{array}\right] .
\end{aligned}
$$

Flux equation can be written as

$$
\left[\begin{array}{c}
\psi_{s M} \\
\psi_{s T} \\
\psi_{r M} \\
\psi_{r T}
\end{array}\right]=\left[\begin{array}{cccc}
L_{s} & 0 & L_{m} & 0 \\
0 & L_{s} & 0 & L_{m} \\
L_{m} & 0 & L_{r} & 0 \\
0 & L_{m} & 0 & L_{r}
\end{array}\right]\left[\begin{array}{c}
i_{s M} \\
i_{s T} \\
i_{r M} \\
i_{r T}
\end{array}\right] .
$$

Torque equation is as follows:

$$
T_{e}=n_{p} L_{m}\left(i_{s T} i_{r M}-i_{s M} i_{r T}\right)
$$

Motion equation can be expressed as

$$
T_{e}=T_{L}+\frac{J}{n_{p}} \frac{d \omega}{d t} .
$$

Secondly, the vector control of induction motor according to rotor flux oriented is described. When the rotor flux orientation is used, $M$ axis coincides with the rotor flux $\psi_{r}$ axis. Since the $T$ axis is perpendicular to the $M$ axis, $\psi_{r}$ on the $T$ axis component is zero, namely, the rotor flux uniquely 
generated by current component on the $M$ axis. So the flux equations are shown as follows:

$$
\begin{gathered}
\psi_{r M}=\psi_{r}=L_{m d} i_{s M}+L_{r d} i_{r M}, \\
\psi_{r T}=0=L_{m d} i_{s T}+L_{r d} i_{r T} .
\end{gathered}
$$

The above equations are converted to the torque equation.

$$
T_{e}=n_{p} \frac{L_{m d}}{L_{r d}} \psi_{r} i_{s T} .
$$

The voltage equation of rotor flux oriented MT coordinate system is given by

$$
\left[\begin{array}{c}
u_{s M} \\
u_{s T} \\
0 \\
0
\end{array}\right]=\left[\begin{array}{cccc}
R_{s}+L_{s} p & -\omega_{1} L_{s} & L_{m} p & -\omega_{1} L_{m} \\
\omega_{1} L_{s} & R_{s}+L_{s} p & \omega_{1} L_{m} & L_{m} p \\
L_{m} p & 0 & R_{r}+L_{r} p & 0 \\
\omega_{s} L_{m} & 0 & \omega_{s} L_{r} & R_{r}
\end{array}\right]\left[\begin{array}{c}
i_{s M} \\
i_{s T} \\
i_{r M} \\
i_{r T}
\end{array}\right]
$$

The induction motor can measure voltage vector, current vector and controlled variable stator; therefore, it is required to find the relationship between each component and other physical measurements of stator side value:

$$
\Psi_{r}=\frac{L_{m d}}{T_{r} p+1} i_{s M},
$$

where $T_{r}=L_{r} / R_{r}$

$$
\omega_{s}=\frac{L_{m d}}{T_{r}} \psi_{r} i_{s T}
$$

The three equations consist of the basic control equations of induction motor vector control system. Also, the realization of control scheme needs motor parameters including $L_{m d}, L_{r d}$, and $T_{r}$, which are closely related to the performance level and accuracy parameters of induction motor control.

\section{Rotor Resistance Influence Factors Analysis}

Main affecting factors to rotor resistance can be regarded as rotor current, running time, frequency, and ambient temperature and can be express as

$$
R_{r}=f(I, t, f, T),
$$

where $R_{r}$ is the rotor resistance, $I$ is the rotor current, $t$ is the running time, $f$ is the frequency, and $T$ is the temperature.

Heating is a dynamic and high inertia nonlinear process; the larger the current and slip frequency are in the longer working time, the more the heat generated. And the temperature caused by this reason combined with ambient temperature determines the rotor temperature. If working time is selected as an input variable, when the motor reaches heat balance, it has no more effect on the rotor resistance change, the 4 factors decrease to 3 , and the other one becomes a disturbance; thus, the accuracy cannot be guaranteed. Considering the rotor resistance change is simply from the temperature rise, winding end temperature can be

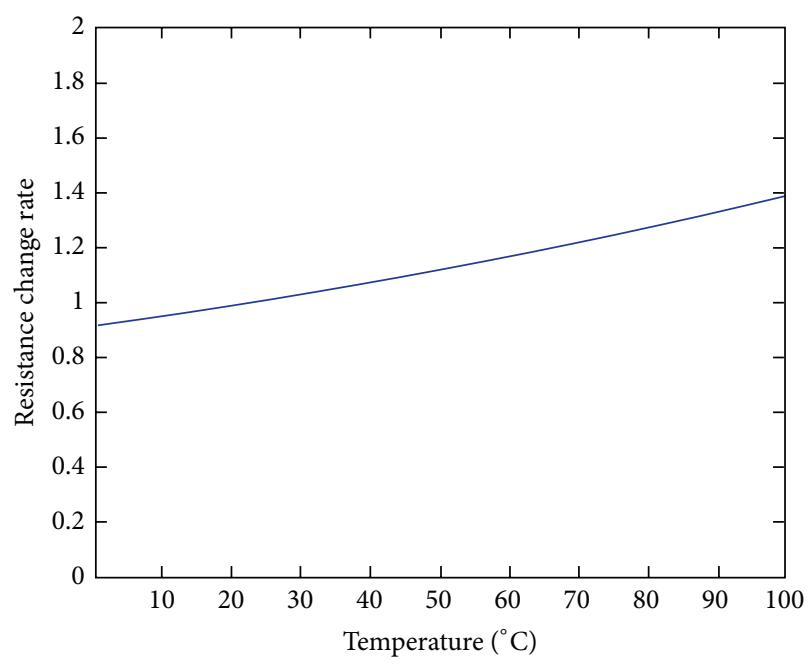

FIGURE 1: Copper wire's resistance change rate with temperature.

selected as a comprehensive input. It also can be used as an important reference of motor fault diagnosis and to realize state monitoring while implementing identification.

The relationship between rotor resistance and temperature is as the following equation:

$$
R_{(T)}=R_{20} \times\left(1+\alpha_{20}(T-20)\right) \text {, }
$$

where $R_{20}$ is copper resistance when ambient temperature is $20^{\circ} \mathrm{C}, R_{(T)}$ is the resistance at temperature $T$, and $\alpha_{20}$ is the temperature coefficient at $20^{\circ} \mathrm{C}$, which is $3.93 \times 10^{-3}$ for copper. Its change rate with temperature is shown in Figure 1.

When the transient value of winding end temperature is constant, rotor resistance also can be affected by other factors, in which the most evident one is slip frequency. When the motor is in stable running state, rotor current's frequency is basically slip resistance, its value is about $1-3 \mathrm{~Hz}$, and the skin effect caused by this reason is very small and can be neglected. When motor is in starting or heavy load process, the skin effect's influence is rather significant, usually 2-3 times of the normal value. According to [23], rotor resistance's change rate with slip frequency can be overfitted with square function as the following equation:

$$
K_{R_{s}}=0.0009 s^{2}+0.0264 s+0.8435 \text {. }
$$

Thus, rotor resistance's change rate with slip frequency is shown in Figure 2.

\section{Elman Neural Network Setup and Parameters Design}

For a given problem, which network structure is selected for its fastest training speed and better output effect is very difficult to predict. It depends on many factors, including the input and output variables, the number of training samples, error target, and the mapping relationship between them. 


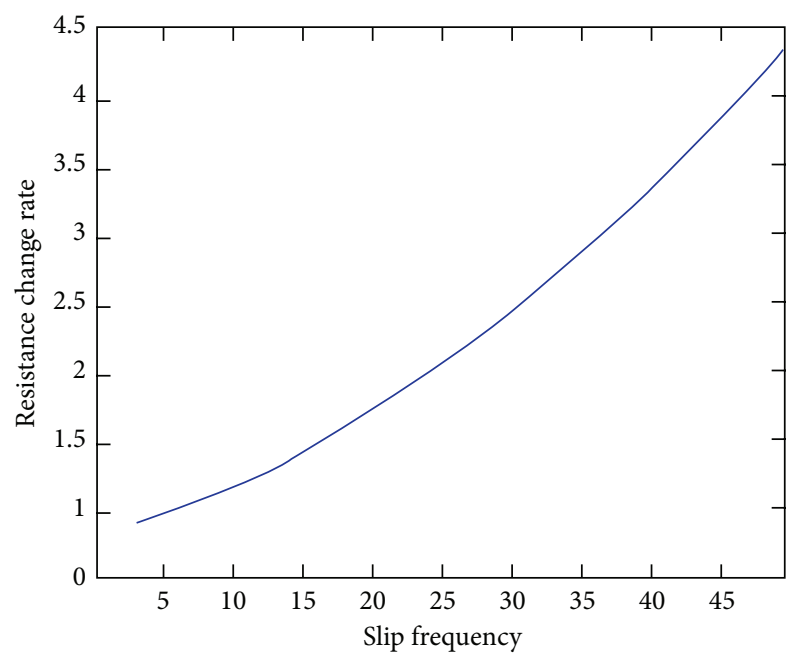

FIGURE 2: Rotor resistance change rate with slip frequency.

4.1. Affiliation Network Structure. The basic Elman neural network structure is shown in Figure 3. Its expression in nonlinear state is

$$
\begin{gathered}
T=\mathrm{TF}^{2} \sum_{j=1, k=1}^{j, k}\left(L W_{j k}^{2} O_{j}^{1}+B_{j k}^{2}\right), \\
O_{j}^{1}=\mathrm{TF}^{1} \sum_{i=1, j=1}^{i, j}\left(I W_{i j}^{1} P_{i}+B_{i j}^{1}+L W_{j j}^{1} D_{j}^{1}\right), \\
D_{j}^{1}(n)=O_{j}^{1}(n-1),
\end{gathered}
$$

where $i, j, k$ is number of input variables, hidden layer neurons, and output layer neurons, respectively; $P, T$ are input and output vectors. $I W_{i j}^{1}, L W_{j k}^{2}$, and $L W_{j j}^{1}$ represent the weights between the number $i$ input and number $j$ hidden layer neuron, the weight between the number $j$ hidden layer neuron and number $k$ output layer neuron, and the weight feedback to the number $j$ hidden layer neuron. $\mathrm{TF}^{1}$, $\mathrm{TF}^{2}$ are transfer functions of hidden layer and output layer, respectively. $b_{i j}^{1}, b_{i k}^{2}$ are bias of the inputs of different layers. $O_{j}^{1}$, $D_{j}^{1}$ are output vector and context layer's feedback state vector.

Weights adjustment function of Elman network has slow convergence speed and may be trapped at local minimum values, and, for specific problem, its default algorithm should be modified; one of the optimized algorithms can be written as

$$
\Delta W^{(n)}=-\eta \frac{\partial e_{p}}{\partial w}+\alpha \Delta W^{(n-1)}
$$

where $\Delta W^{(n)}$ is revised value at $n$ iteration, $\eta$ is acceleration factor, and $\alpha$ is momentum factors. According to research, when $\eta=0.2-0.5, \alpha=0.9-0.98$, the algorithm has better convergence speed and can avoid beating back and forth when error curve face is long and narrow.

Since the input data fluctuation range is very large, when they are used as input directly, the larger input data often blurs smaller input data, which causes training time to increase greatly while precision of the network output drops. Therefore, in order to avoid the inherent characteristics of data loss, it needs to have the input normalized for the sake of better training and simulation performance. Training performances of the network before and after normalization are shown in Figure 4.

Figure 4(a) shows that, before normalization, error drops quickly in the first 100 epochs, but its change rate becomes very slowly; thereafter, its error failed to reach $1 \%$ at maximum points of 1000 epochs. Figure 4(b) shows that, after normalization, training speed of the network increases significantly and reaches the training goal of $0.1 \%$ accuracy at epoch 102. It is clear that a reasonable normalization parameter is of great influence on the convergence rate and the training time.

4.2. Training Data Processing. The complexity of motor parameters identification is determined because of the multivariable, nonlinear, and strong coupling characteristics of its mathematical model. The relationship between the parameters to be identified with the motor running state variables is difficult to be expressed by functions, some influence factors even not to be fully studied. So it is started with factors that have clear relationship with the input variable, and, after its effects are compared and verified, other potential influence factors may then be gradually added and analyzed.

In order to ensure neural network's better response to various inputs, the completeness of training samples is required. Samples data acquirement must be in consideration of various operation conditions of the motor in actual circumstances, and its capacity should be large enough to include all the characteristics of the target system.

Here, winding end temperature $T$, slip frequency $S$, and rotor current $I$ are selected as the main conditions, and, after variables combination of the input, abundant motor running state is covered.

In the classical or improved motor parameter testing method, acquisition of training data and network validation data is very time consuming and almost cannot be completed. Some methods can be applied to maximize the use of limited data. A reliable way is to obtain a batch of training samples with the traditional methods, and then a reference rotor resistance model is set up according to the data. In MATLAB/Simulink, there are several parameters that can be set in 3-phase asynchronous motor model; thus, a lot of network training data can be acquired in simulation combining with the established rotor resistance model.

At moment $i$, rotor resistance is not only affected by the external environment and internal factors, but also affected by history value, such as the value at moment $i-1, i-2, \ldots$, and $i-n$. If the past $N$ data are used to observe the value in the future $M$ times, the $N$ adjacent samples data are selected as the sliding window and mapped to $M$ values. A certain overlapping samples data segment is designed. Its principle is in Figure 5.

In the process of normalization, maximum value selection is involved. Temperature value can be selected in reference to motor's insulation class detailed in Table 1. 


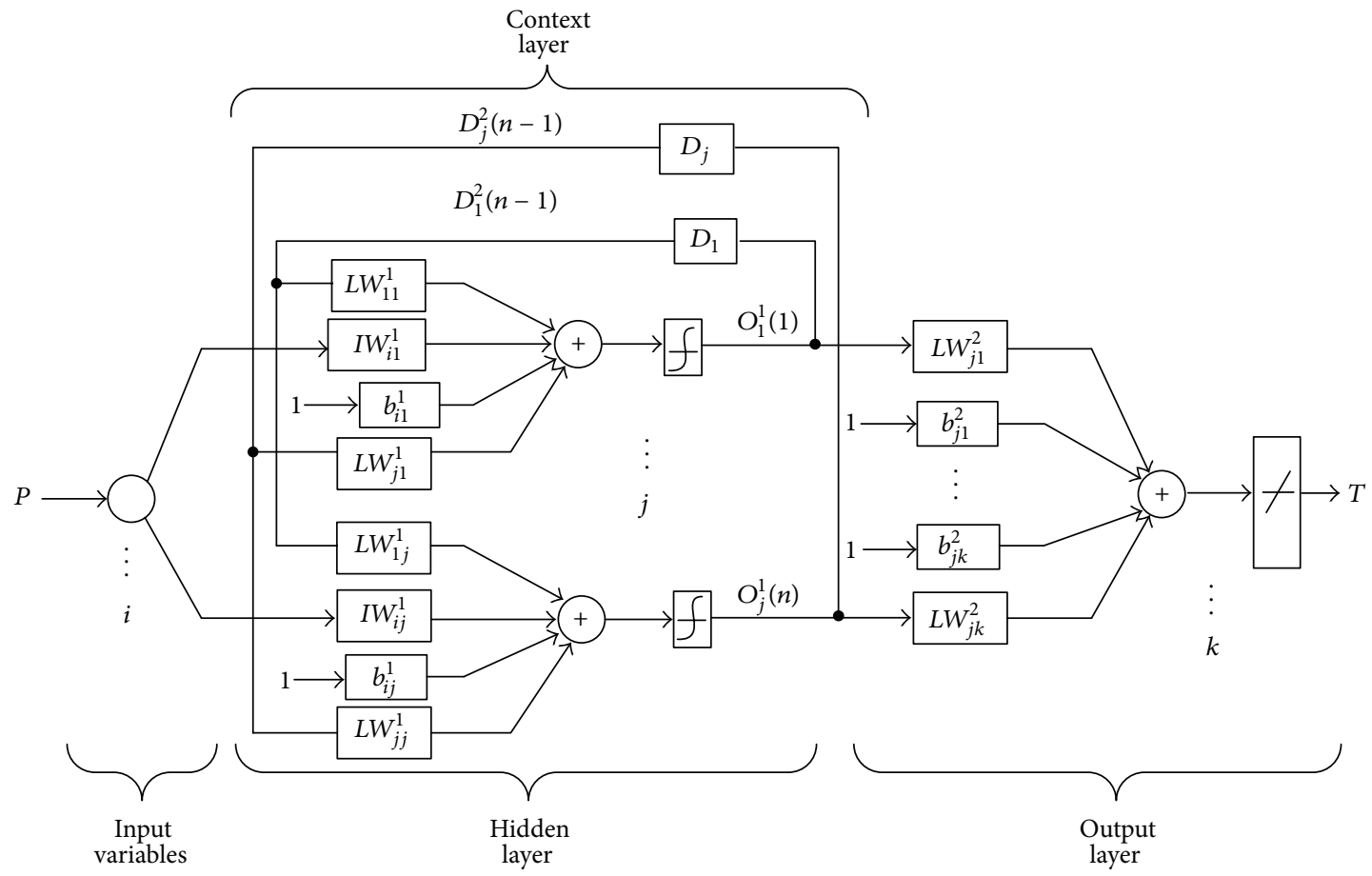

FIGURE 3: Elman neural network structure.

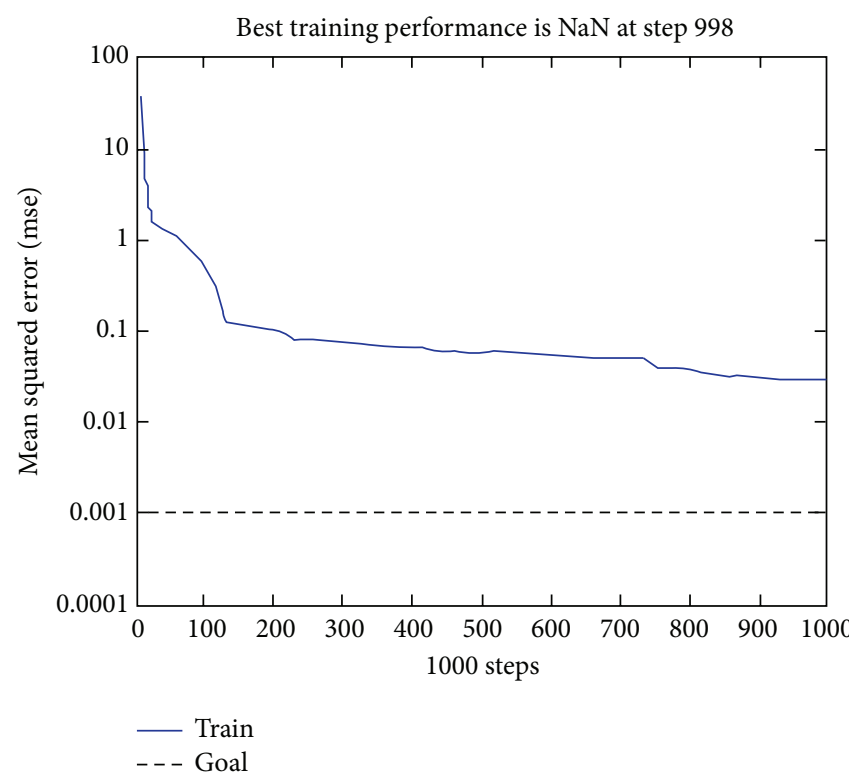

(a) Before normalization

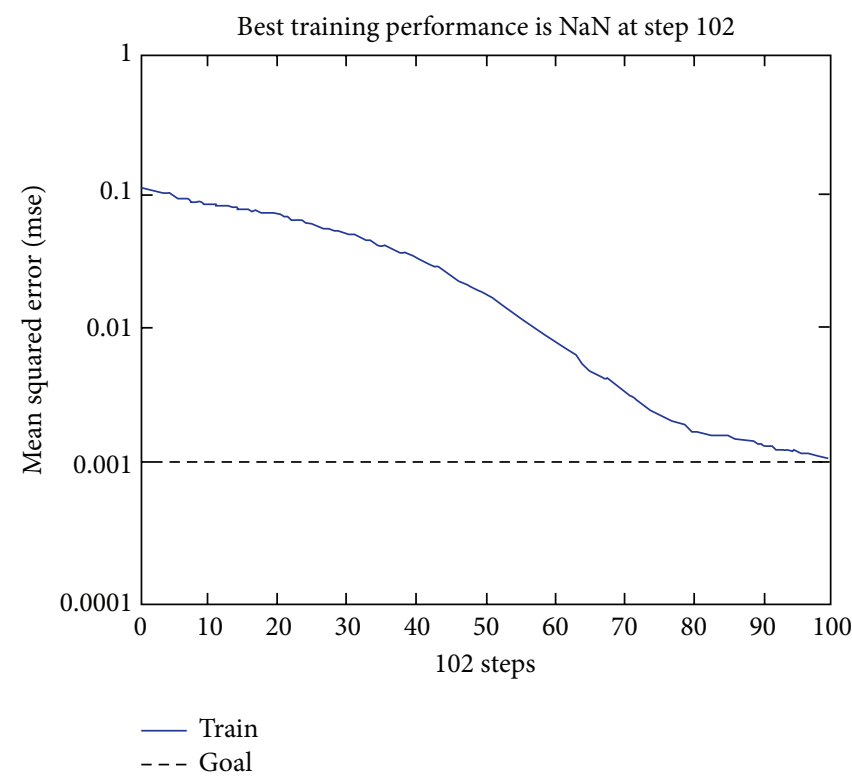

(b) After normalization

FIGURE 4: Training performance of the network with normalization.

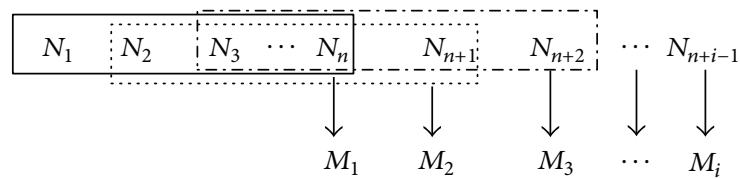

FIgURE 5: Slide window sampling.
TABLE 1: Motor's insulation class and maximum degree rise.

\begin{tabular}{lccccccc}
\hline $\begin{array}{l}\text { Insulation } \\
\text { class }\end{array}$ & $\mathrm{Y}$ & $\mathrm{A}$ & $\mathrm{E}$ & $\mathrm{B}$ & $\mathrm{F}$ & $\mathrm{H}$ & $\mathrm{C}$ \\
\hline $\begin{array}{l}\text { Max. degree } \\
\text { rise }\end{array}$ & 90 & 105 & 120 & 130 & 155 & 180 & 220 \\
\hline
\end{tabular}




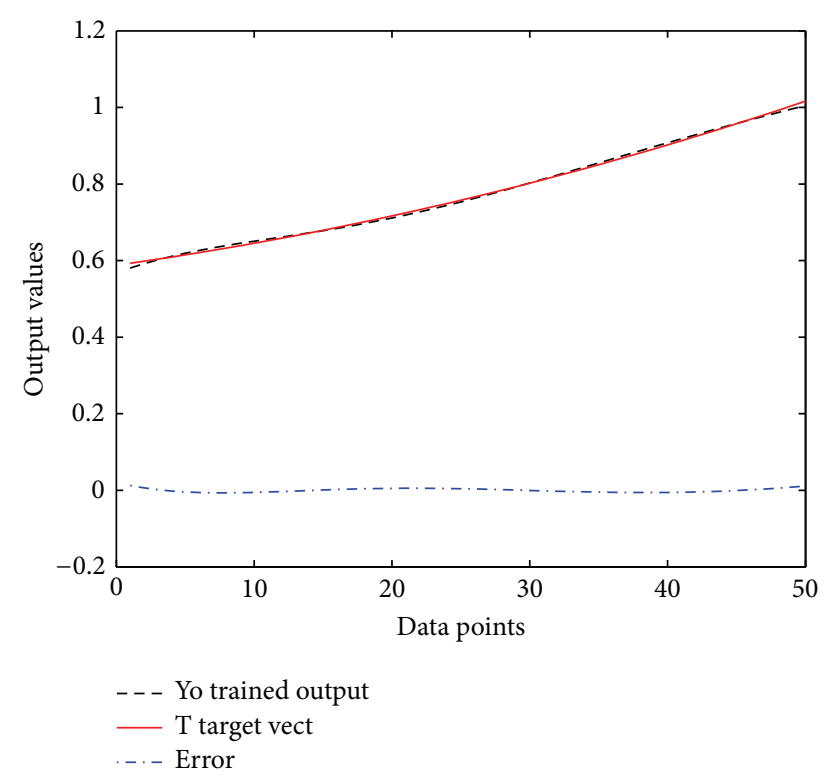

FIGURE 6: Rotor resistance 2 input network simulation and error curve.

TABLE 2: Training steps of different hidden layer neurons.

\begin{tabular}{lccccc}
\hline Hidden layer neurons & 5 & 6 & 7 & 8 & 9 \\
\hline Training steps & 180 & 138 & 120 & 137 & 141 \\
\hline
\end{tabular}

Usually the maximum current of induction motor appears in starting state, and the biggest starting current is about 4-7 times of its rating value. Maximum slip frequency usually appears at starting moment that is a transient process, so maximum slip frequency during motor running process is selected. Here the normalized parameter for temperature is 50 , slip frequency is 25 , and rotor resistance is 5 .

\section{Rotor Resistance Identification with Elman Neural Network}

At first, the rotor temperature and slip frequency are used as the input parameters of Elman neural network to check up the effect of this network. Then, the rotor current is added as the input parameter to make sure whether the identification results have improved or not with this network, and the number of input parameters in this network is determined at last.

For prominent influence factor, its inner nonlinear mapping relationship is strong, number of Elman hidden layer neurons may be small, and the relationship between it and training steps is shown in Table 2.

As the number of hidden layer neurons increases, the training steps have not been reduced, but reduced to a certain point in which these steps have a tendency to increase. A reasonable explanation is that, after the structure of the network completes the nonlinear mapping from input to output, the increase of the neurons number leads to increase of the weights and feedbacks need to adjust. In order to

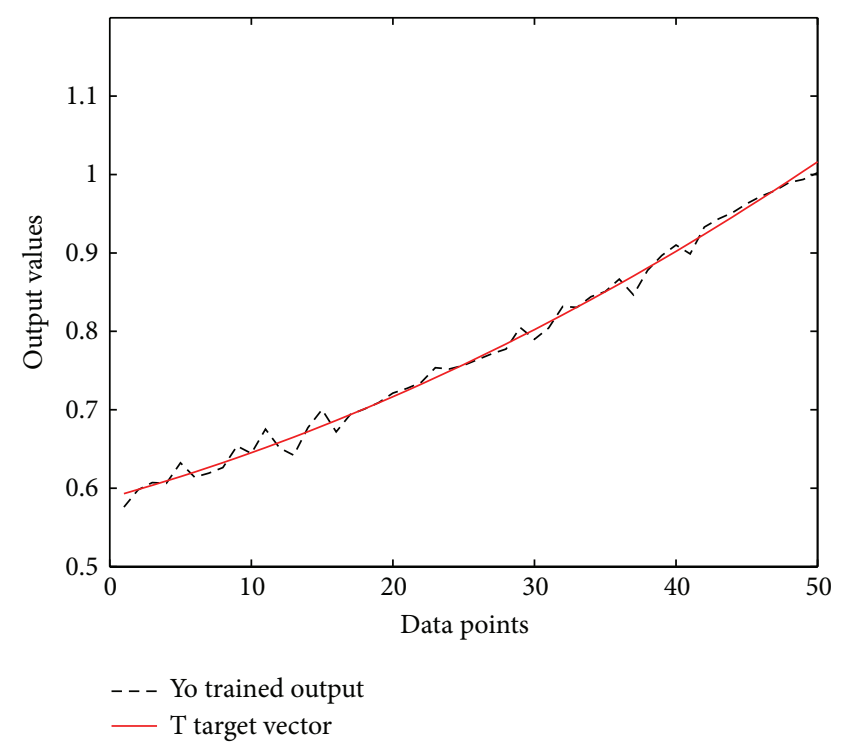

FIGURE 7: Rotor resistance 3 input network simulation curve.

achieve the same error level, quantity and complexity of the calculation increase dramatically, so it needs more steps to adjust. The increased neurons are redundant in some degree, though robustness of the network increases, but it is not desirable for the contemporary processor and electronic devices realization. Here, 2-7-1 network structure is selected.

In Figure 6, it shows that the constructed network has good performance to the input, and the error in most data points is below $1 \%$. At the last 5 points, the error increases. In particular at point 50 , it reaches $2 \%$, which is large enough to have certain effect on the high control performance, but these data points represent transient starting period, and its error is acceptable to the overall control performance.

When current is added as input, the nonlinear mapping relationship between input and output become more complicated, and network size should be increased. Started with 7 as hidden layer neurons and with comprehensive consideration of train performance, the hidden layer neurons number is selected as 10. As shown in Figure 7, error levels at most data points are bigger than $\pm 1 \%$, and beyond $3 \%$ at the last several points.

\section{Simulation and Experiment Results Analysis}

An experimental platform is built and shown in Figure 8. The rotor resistance parameter identification based on Elman neural network is applied to the induction motor inverter.

In this platform, the parameters of asynchronous motor are $P_{N}=4 \mathrm{~kW}, U_{N}=380 \mathrm{~V}, N_{p}=2, R_{s}=1.405 \omega, R_{r}=$ $1.395 \omega, L_{s}=5.83 \mathrm{mH}, L_{r}=5.84 \mathrm{mH}, L_{m}=172 \mathrm{mH}$, and $T_{r}=0.138$.

At first, the simulation platform is designed with MATLAB/Simulink. The motor parameter $R_{r}$ (rotor resistance) is identified with the traditional offline method and Elman neural network online method, respectively. The comparison with the two methods is shown in Figure 9. 


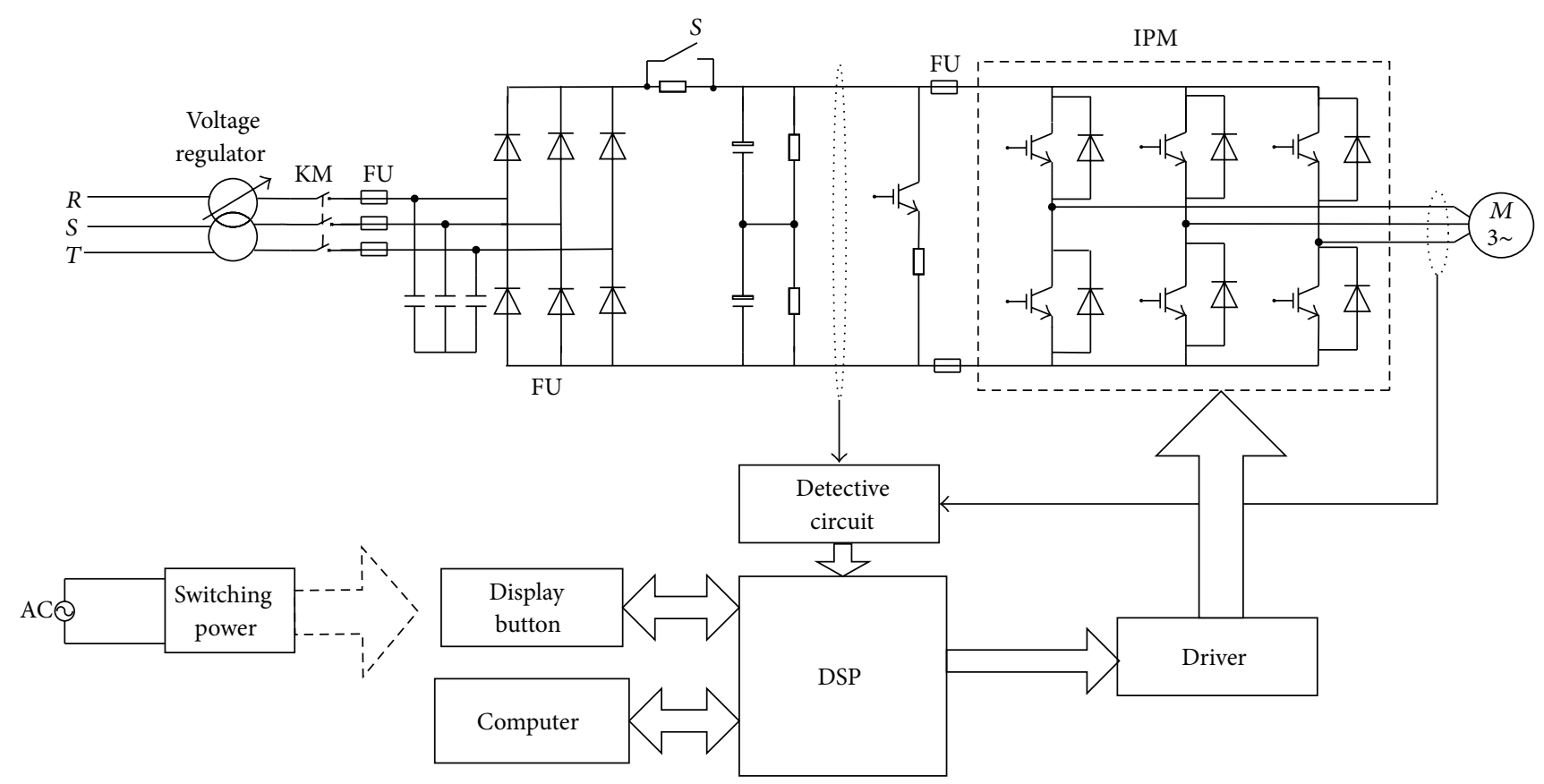

(a) Induction motor inverter system diagrams

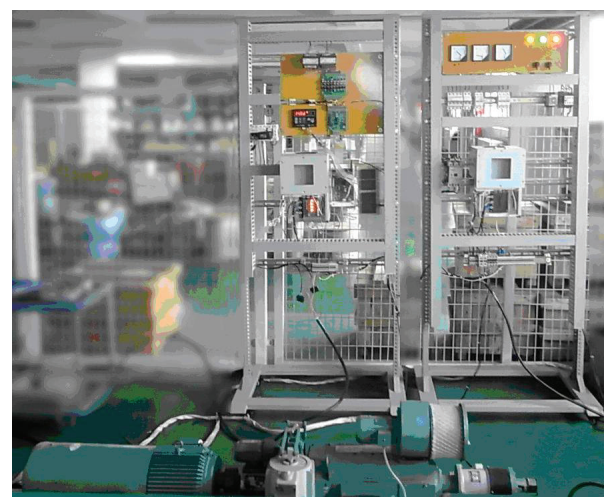

(b) Experimental hardware photo

FIGURE 8: Induction motor inverter experimental platform.

It is obvious that the identification result with traditional offline method cannot adapt to the temperature rise and the slip frequency change. Its ability is not enough to adapt to the requirement of high performance of the control system. However, Elman neural network online identification method is a very good solution. It also has the similar results for the parameter $T_{r}$ identification.

The simulation for the variation of motor parameters is accomplished with the Simulink-based motor model, shown in Figure 10. The cures of the flux linkage and torque are obtained before and after the parameters' change. Curves show that parameters' change has a great influence on the performance of motor control system based on the steady-state mathematical model. If these motor parameters can be updated real-time and used in the control system, the control accuracy will be improved to a large extent.
It can be seen from Figure 10 that the torque estimation is to reach a stable value about 0.4 seconds with the given torque starting in 50 . When the calculation model is corresponding to motor actual parameters, torque ripple initially reaches around $200 \%$, but it soon becomes normal value and more smooth. However, if the torque estimation model parameters are different from the motor parameters, namely, these parameters change during motor running process and the estimation model fails to update them, the torque reaches more than $450 \%$ with large fluctuation. Moreover, even if it is stable at last, there is still big fluctuation about the true value of $15 \%$.

In Figure 11, it shows that the motor rotor resistance change has effect on the flux observation. When the observation model adopts the accurate motor parameters, the observed flux and the measurement output are almost the same, which is better to tracking the motor flux. When the 


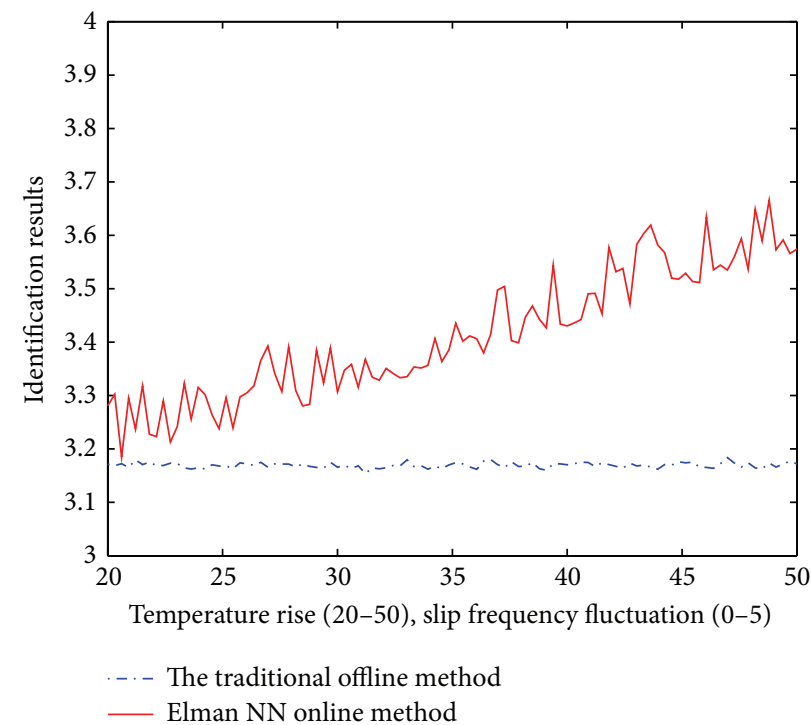

FIGURE 9: Identification results comparison with traditional offline method and Elman NN online method.

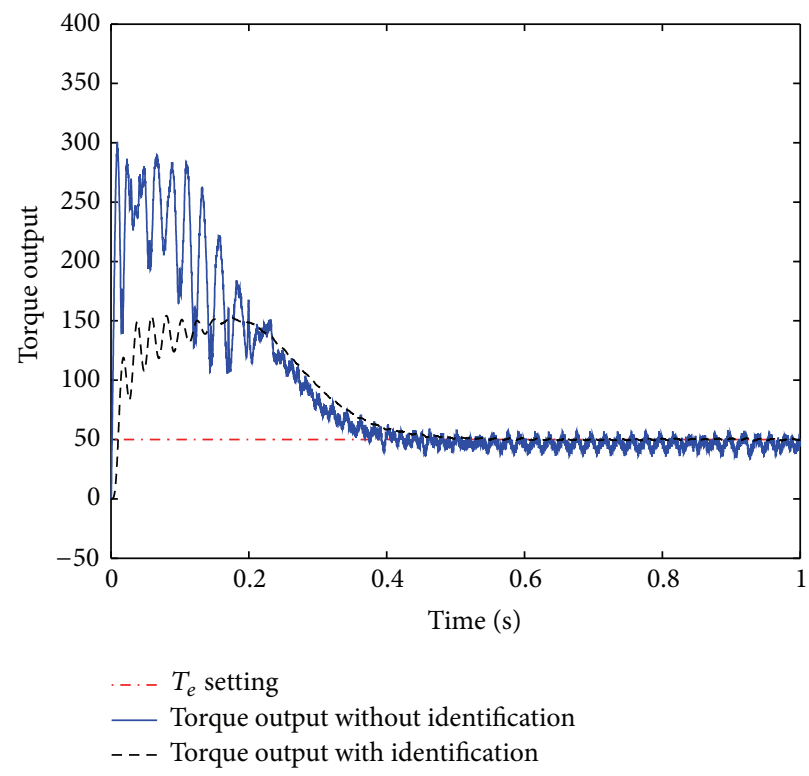

FIgURE 10: Torque output before and after parameter change.

rotor resistance value is double, the observed flux has large fluctuation at the motor starting, which is beyond the exact value of nearly $200 \%$ and tends to accurate value at the speed stability with small fluctuation. When the rotor resistance value is triple, the fluctuation of the observed flux is still large; it is obvious at stable phase, about 3.5\%. When the rotor resistance value is increased 5 times, the observed flux has the obvious fluctuation that reaches about $6.7 \%$. This error is a great obstacle to improve the precision of the control system.

In Figure 12, the experimental results are shown including inverter output voltage and current with rotor resistance parameter identification. From these results, it is obvious that the accuracy of the motor parameter identification plays an important role to improve the performance in adjustable speed drive system.

\section{Conclusion}

In this paper, a method on induction motor parameter online identification based on Elman neural network is proposed. As a feedback neural network, its abilities of nonlinear function approximation and time-varying adaptivity are used to resolve the rotor resistance identification problems. According to various running conditions with the realistic environment, the influence factors of this parameter are analyzed to ensure the completeness of the training samples.

Simulation and experimental results show that this method has the higher applicability of identification of induction motor parameters. With the experiment of the variation of rotor resistance, the torque curves of the before and after parameter changes are obtained, which show that the 


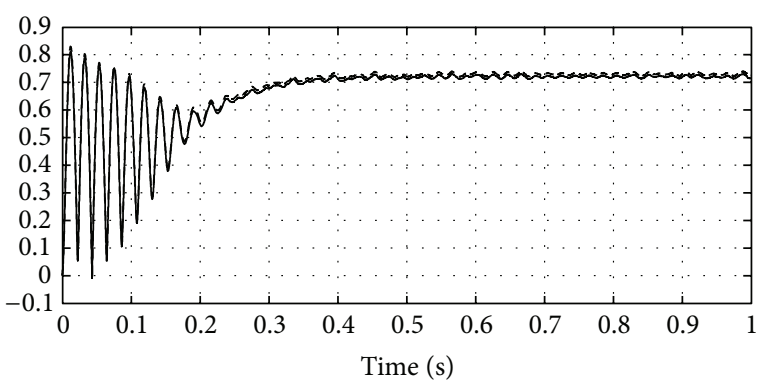

(a) Resistance value is accurate

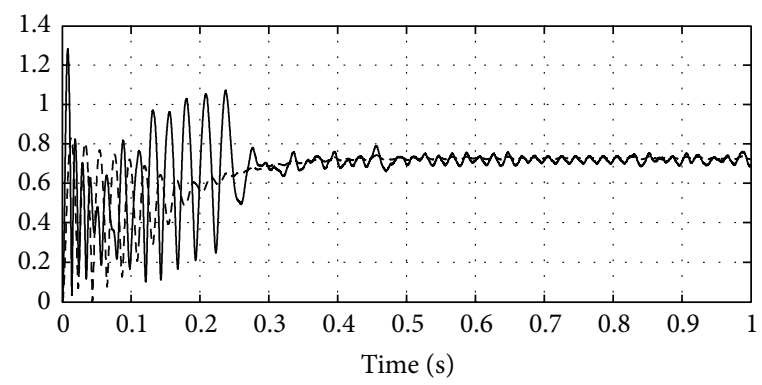

(c) Resistance value is triple

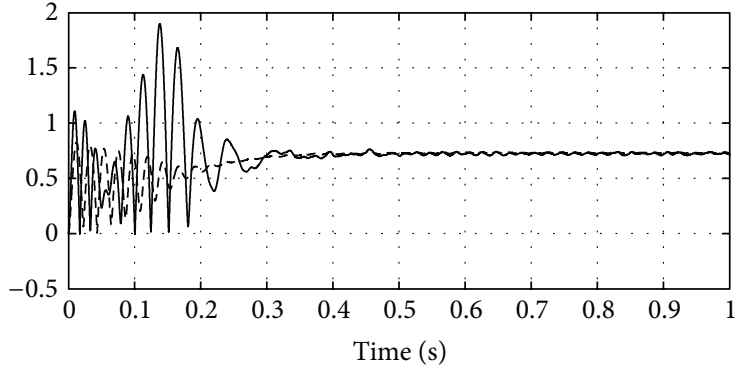

(b) Resistance value is double

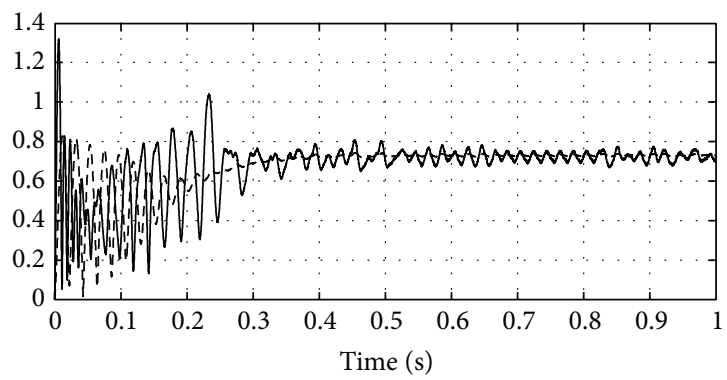

(d) Resistance value is increased by 5 times

FIGURE 11: Rotor flux comparison with different rotor resistance values.

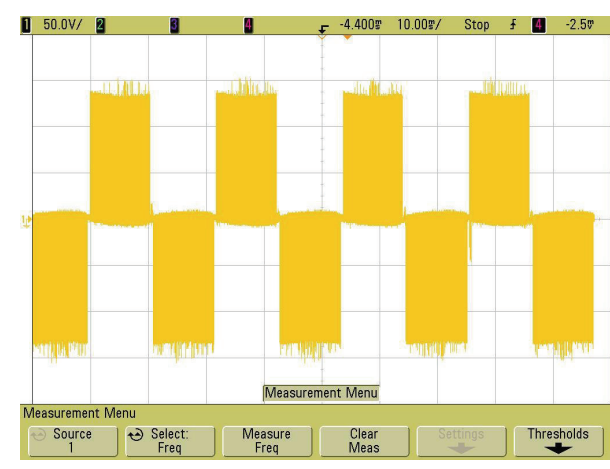

(a) Output voltage waveform

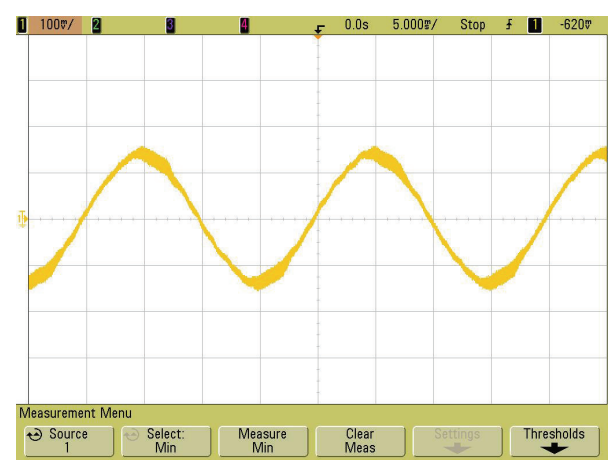

(b) Output current waveform

FIGURE 12: Experimental outputs including rotor resistance identification.

performance of vector controlled induction motor with the steady-state mathematical model is influenced by parameter changes remarkably. The output voltage and current curves, which were obtained from the experimental platform, show that the vector control with rotor resistance identification has improved performance.

\section{Conflict of Interests}

The authors declare that there is no conflict of interests regarding the publication of this paper.

\section{Acknowledgments}

The authors would like to thank the funding support by the University of Macau, Grant no. MYRG079(Y1-L2)-FST13YZX. Additionally, this work is supported by the Key
Program of Educational Commission in Henan Province of China (13A470246) and the Innovation Science Foundation of Henan University of Science and Technology (2013ZCX019).

\section{References}

[1] S. Bolognani, L. Peretti, and M. Zigliotto, "Parameter sensitivity analysis of an improved open-loop speed estimate for induction motor drives," IEEE Transactions on Power Electronics, vol. 23, no. 4, pp. 2127-2135, 2008.

[2] Y. Fan, W.-L. Qu, H.-F. Lu et al., "Slip frequency correction method base on rotor flux $\mathrm{q}$ axis component for induction machine indirect vector control system," Proceedings of the CSEE, vol. 29, no. 9, pp. 62-66, 2009.

[3] H. A. Toliyat, E. Levi, and M. Raina, "A review of RFO induction motor parameter estimation techniques," IEEE Transactions on Energy Conversion, vol. 18, no. 2, pp. 271-283, 2003. 
[4] E. Foulon, C. Forgez, and L. Loron, "Resistances estimation with an extended Kalman filter in the objective of real-time thermal monitoring of the induction machine," IET Electric Power Applications, vol. 1, no. 4, pp. 549-556, 2007.

[5] L.-C. Zai, C. L. DeMarco, and T. A. Lipo, "An extended Kalman filter approach to rotor time constant measurement in PWM induction motor drives," IEEE Transactions on Industry Applications, vol. 28, no. 1, pp. 96-104, 1992.

[6] A. Accetta, M. Cirrincione, M. Pucci, and G. Vitale, "Neural sensorless control of linear induction motors by a full-order Luenberger observer considering the end-effects," in Proceedings of the 4th Annual IEEE Energy Conversion Congress and Exposition (ECCE '12), pp. 1864-1871, IEEE, September 2012.

[7] S. M. N. Hasan and I. Husain, "A Luenberger-sliding mode observer for online parameter estimation and adaptation in high-performance induction motor drives," IEEE Transactions on Industry Applications, vol. 45, no. 2, pp. 772-781, 2009.

[8] K.-B. Lee and F. Blaabjerg, "Reduced-order extended Luenberger observer based sensorless vector control driven by matrix converter with nonlinearity compensation," IEEE Transactions on Industrial Electronics, vol. 53, no. 1, pp. 66-75, 2006.

[9] R. Blasco-Gimenez, G. Asher, M. Sumner, and K. Bradley, "Dynamic performance limitations for MRAS based sensorle ss induction motor drives. Part 1: stability analysis for the closed loop drive," IEE Proceedings-Electric Power Applications, vol. 143, no. 2, pp. 113-122, 1996.

[10] S. Maiti, C. Chakraborty, Y. Hori, and M. C. Ta, "Model reference adaptive controller-based rotor resistance and speed estimation techniques for vector controlled induction motor drive utilizing reactive power," IEEE Transactions on Industrial Electronics, vol. 55, no. 2, pp. 594-601, 2008.

[11] M. Cirrincione, A. Accetta, M. Pucci, and G. Vitale, "MRAS speed observer for high-performance linear induction motor drives based on linear neural networks," IEEE Transactions on Power Electronics, vol. 28, no. 1, pp. 123-134, 2013.

[12] Y. Shi, K. Sun, L. Huang, and Y. Li, "Online identification of permanent magnet flux based on extended Kalman filter for IPMSM drive with position sensorless control," IEEE Transactions on Industrial Electronics, vol. 59, no. 11, pp. 4169-4178, 2012.

[13] G. Kenné, R. S. Simo, F. Lamnabhi-Lagarrigue, A. Arzandé, and J. C. Vannier, "An online simplified rotor resistance estimator for induction motors," IEEE Transactions on Control Systems Technology, vol. 18, no. 5, pp. 1188-1194, 2010.

[14] G. Kenné, T. Ahmed-Ali, F. Lamnabhi-Lagarrigue, and A. Arzandé, "Nonlinear systems time-varying parameter estimation: application to induction motors," Electric Power Systems Research, vol. 78, no. 11, pp. 1881-1888, 2008.

[15] C.-H. Fang, S.-K. Lin, and S.-J. Wang, "On-line parameter estimator of an induction motor at standstill," Control Engineering Practice, vol. 13, no. 5, pp. 535-540, 2005.

[16] S. Du, Y. Shen, and Z. Ji, "Research on induction motor resistance identification based on FMRAS," Small \& Special Electrical Machines, vol. 36, no. 10, pp. 51-54, 2008.

[17] L. Dong, Y. Li, W. Wang, and M. Li, "Rotor resistance adaptive identification for vector control of induction motors," Transactions of China Electrotechnical Society, vol. 17, pp. 14-33, 2002.

[18] B. K. Bose, "Neural network applications in power electronics and motor drives-an introduction and perspective," IEEE Transactions on Industrial Electronics, vol. 54, no. 1, pp. 14-33, 2007.

[19] G. Sheng-wei, W. You-Hua, C. Yan, and Z. Chuang, "Design and simulation of artificial-neural-network-based rotor resistance observer of induction motors," in Proceedings of the 2nd International Conference on Intelligent Networks and Intelligent Systems (ICINIS '09), pp. 593-596, Tianjin, China, November 2009.

[20] L. Wang, G. X. Zhou, and Q. D. Wu, "Hopfield neural network based linear system parameter identification scheme and its application in asynchronous motor drive system identification," Proceedings of the Chinese Society of Electrical Engineering, vol. 21, no. 3, pp. 9-11, 2001.

[21] L. Wang, G. Wang, and W. Ma, "A new method for parameters identification of synchronous electric machine based on wavelet transform and neural network," Chinese Society for Electrical Engineering, vol. 27, no. 3, pp. 1-6, 2007.

[22] W. Song, S.-S. Shi, C. Chao, Y. Gang, and Z.-J. Qu, "Identification of PMSM based on EKF and elman neural network," in Proceedings of the IEEE International Conference on Automation and Logistics (ICAL '09), pp. 1459-1463, Shenyang, China, August 2009.

[23] X. Wu and X. Wang, "Calculation of skin effect for double-cage rotor bar of the induction machine," Proceedings of the CSEE, vol. 23, no. 8, pp. 116-120, 2003. 


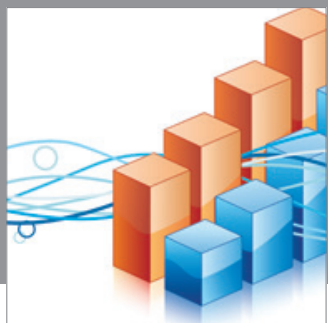

Advances in

Operations Research

mansans

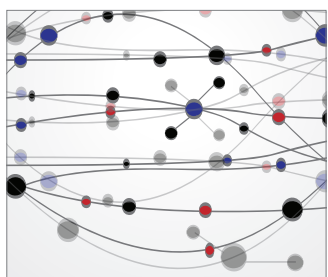

The Scientific World Journal
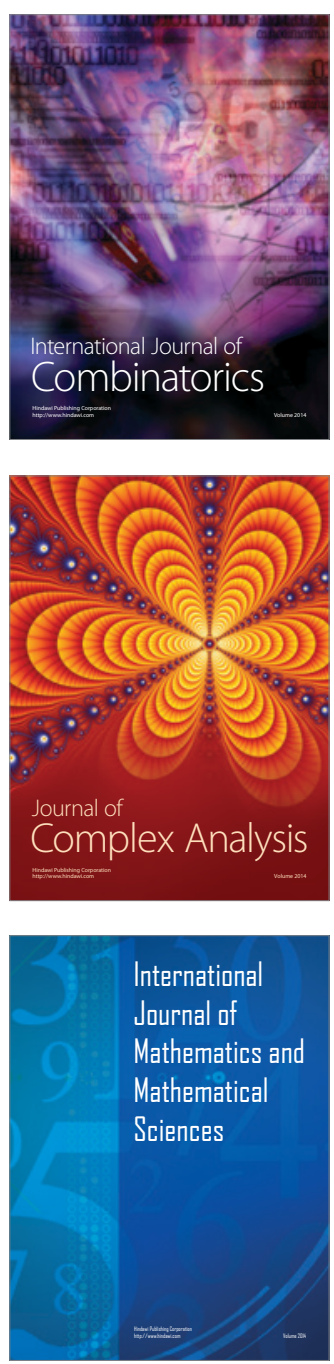
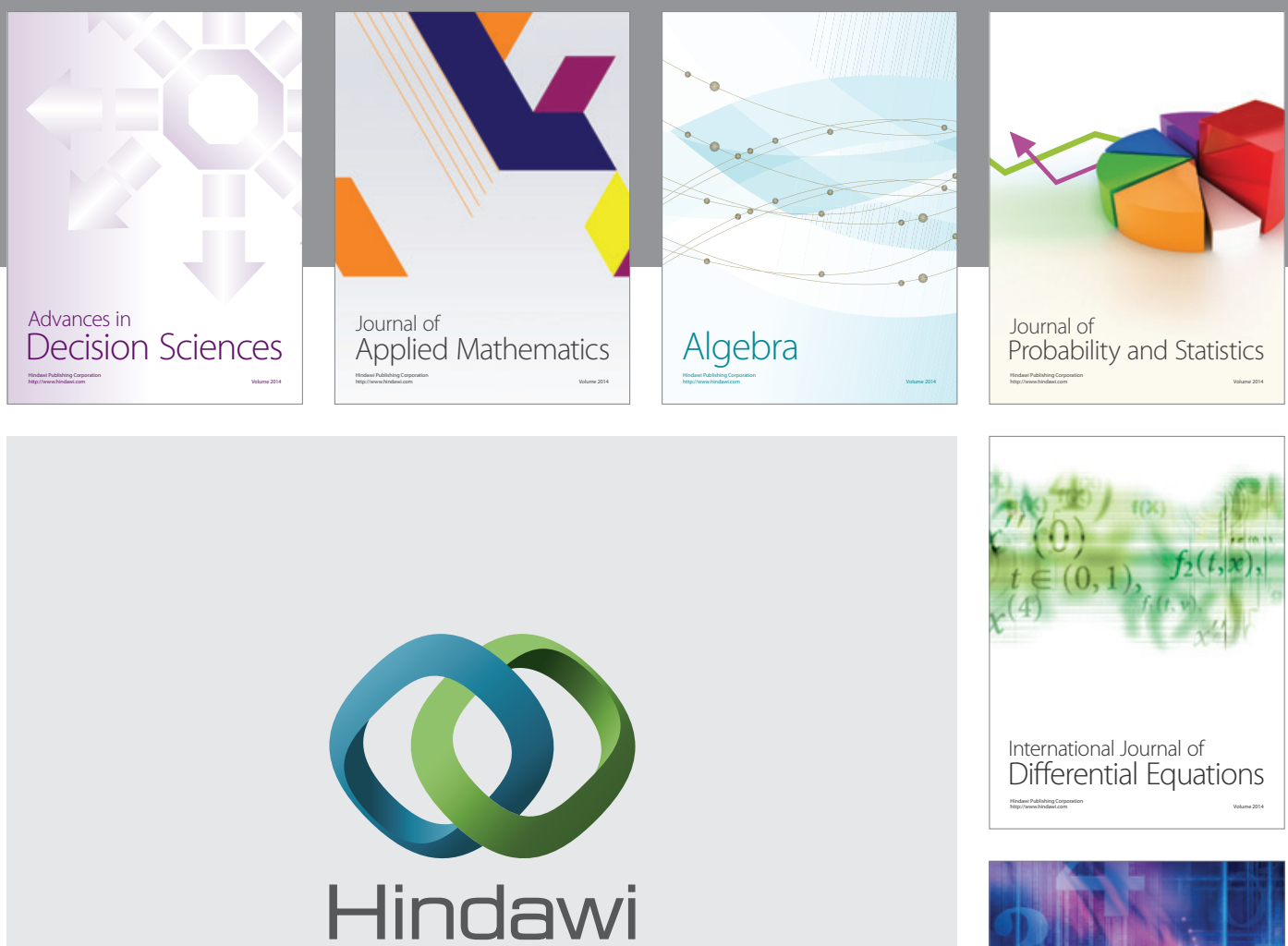

Submit your manuscripts at http://www.hindawi.com
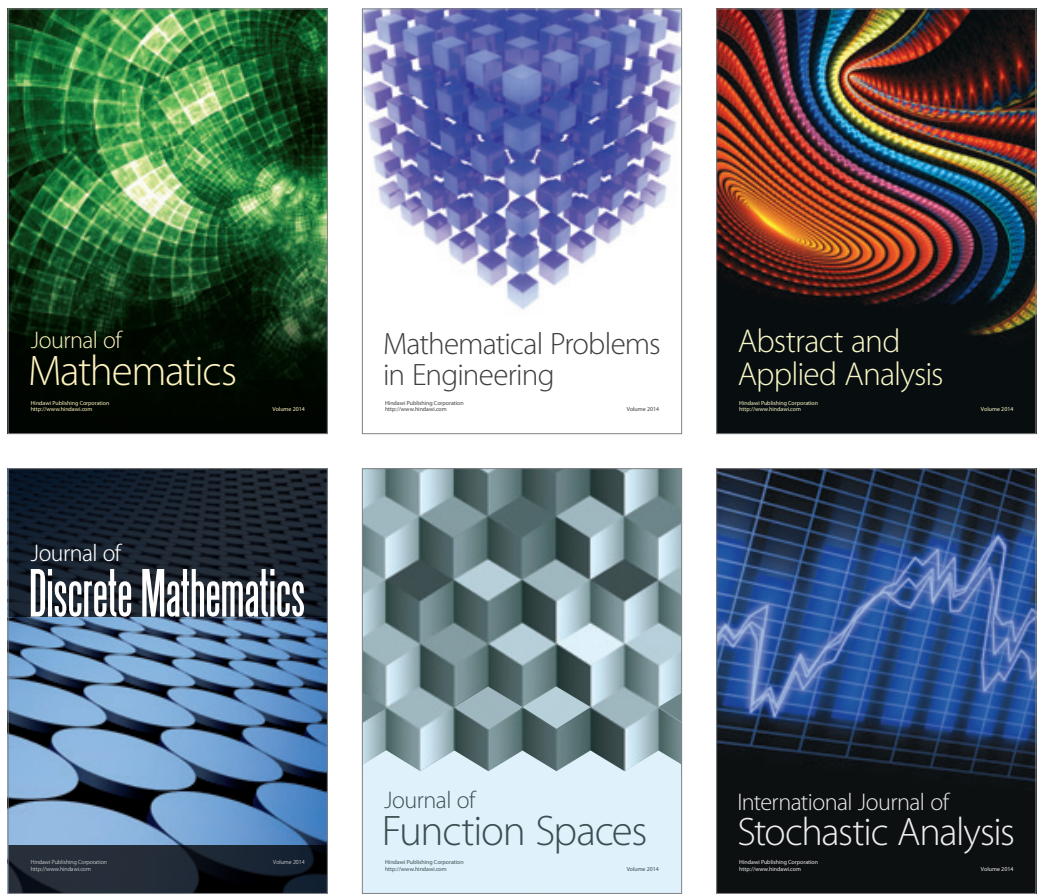

Journal of

Function Spaces

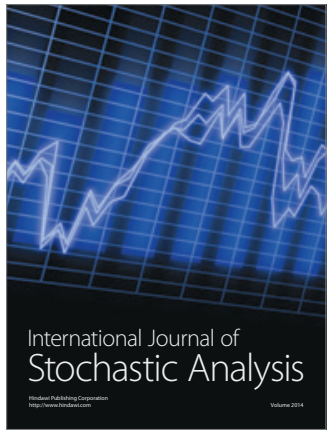

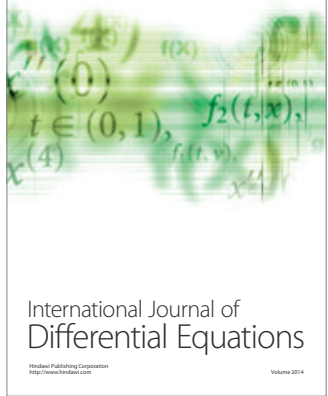
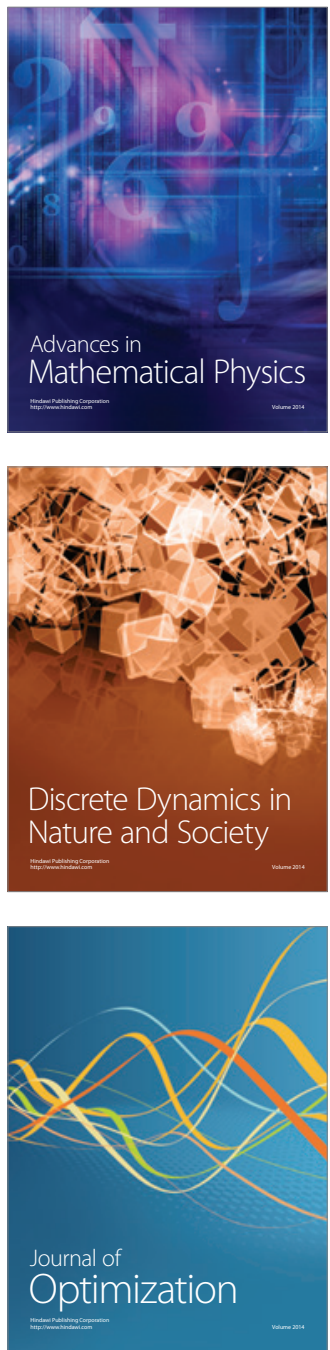